\title{
A Training System for the MyoBock hand in a Virtual Reality Environment
}

\author{
Go Nakamura ${ }^{1,2,3}$, Taro Shibanoki ${ }^{4}$, Keisuke Shima ${ }^{5}$, Yuichi Kurita ${ }^{4}$, Masaki Hasegawa ${ }^{6}$, \\ Akira Otsuka $^{6}$, Yuichiro Honda ${ }^{1,2}$, Takaaki Chin $^{7}$ and Toshio Tsuji ${ }^{4}$ \\ ${ }^{1}$ Robot Rehabilitaion Center in Hyogo Rehabilitaion Center, Kobe, 651-2181 Japan \\ ${ }^{2}$ The Hyogo institute of Assistive Technology, Kobe, 651-2181 Japan \\ ${ }^{3}$ Graduate School of Engineering, Hiroshima University, Higashi-hiroshima, 739-8527 Japan \\ ${ }^{4}$ Institute of Engineering, Hiroshima University, Higashi-hiroshima, 739-8527 Japan \\ ${ }^{5}$ Faculty of Engineering, Yokohama National University, Yokohama, 240-8501 Japan \\ ${ }^{6}$ Faculty of Health and Welfare, Prefectural University of Hiroshima, Mihara, 723-0053 Japan \\ ${ }^{7}$ Department of Orthopaedic Surgery, Hyogo Rehabilitation Center Hospital, Kobe, 651-2181 Japan
}

\begin{abstract}
This paper proposes a novel EMG-based MyoBock training system that consistently provides a variety of functions ranging from EMG signal control training to task training. Using the proposed training sytem, a trainee controls a virtual hand (VH) in a 3D virtual reality (VR) environment using EMG signals and position/posture information recorded from the trainee. The trainee can also perform tasks such as holding and moving virtual objects using the system. In the experiments of this study, virtual task training developed with reference to the Box and Block Test (BBT) used to evaluate myoelectric prostheses was conducted with two healthy subjects, who repeatedly performed 10 one-minute tasks involving grasping a ball in one box and transporting it to another. The BBT experiments were also conducted in a real environment before and after the virtual training, with results showing an improvement in the number of tasks successfully completed. It was therefore confirmed that the proposed system could be used for myoelectric prosthesis control training.
\end{abstract}

\section{INTRODUCTION}

Myoelectric prostheses [1]-[4] such as MyoBock (Otto Bock Corp.) are provided to help normalize life for upper-limb amputees. To achieve free control of prostheses in daily life, it is necessary to secure voluntary generation of EMG signals and conduct task training using the prosthesis under the instruction of doctors's and therapists's over a period of a few months [5]. In this context, EMG control training systems have been proposed [6]-[9] to support the recovery of muscle function and promote voluntary muscle contraction. For example, Tsuji et al. [6] proposed an EMG-based rehabilitation aid (EBRA) that supports three types of EMG-based training for voluntary muscular contraction, collaboration among multiple muscles and muscular contraction timing. However, these systems focus only on training for voluntary control of EMG signals, and do not support myoelectric prosthesis control training. In the use of prostheses, EMG signals vary with changes in arm position/posture and the generation of force to support the weight of the prosthesis. Accordingly, even if voluntary control of EMG signals can be achieved through training, it can still be difficult for user to perform target tasks using a prosthesis. For this reason, extensive training with a myoelectric unit is necessary before the user can control the prosthesis freely in daily life. However, as myoelectric prostheses are very expensive, medical facilities often neither have them nor can offer long-term training in their operation.

Against this background, a system to support task training with myoelectric prostheses using virtual reality has been developed [10]-[15]. Hauschild et al. [14] and Lambrecht et al. [15] proposed a system in which subject/patients can operate a simulated limb and hold/release objects with it in a virtual environment. However, trainees cannot conduct task training without EMG signal control ability.

This paper proposes a MyoBock training system that consistently provides a variety of functions ranging from EMG signal control training to task training with the aim of supporting MyoBock prosthetic hand prescription.

\section{Proposed Training System}

The proposed system involves the two stages of EMG signal control training and task training, which are outlined below.

\section{A. EMG Signal Control Training [6]}

The EMG signal control training to recover muscular function and promote myoelectric prosthesis control ability is conducted using the EBRA method proposed by Tsuji et al. [6]. The EBRA approach allows three types of EMG-based training for voluntary muscular contraction, collaboration among multiple muscles and muscular contraction timing. In contraction training, trainees are instructed to maintain a target level of muscular contraction to build their ability in generating EMG signals. In collaboration training, trainees practice controlling the contraction level of each muscle by matching the EMG pattern for the target motion as extracted in real time. In timing training, trainees are instructed on the timing of desired motions and try to reproduce them accordingly. It is possible to modify difficulty and other parameters depending on the trainee's EMG signal control skill in these three types of training. Task training is subsequently performed after the trainee has acquired the ability to control EMG signals. The details of the proposed part of task training are outlined below. 


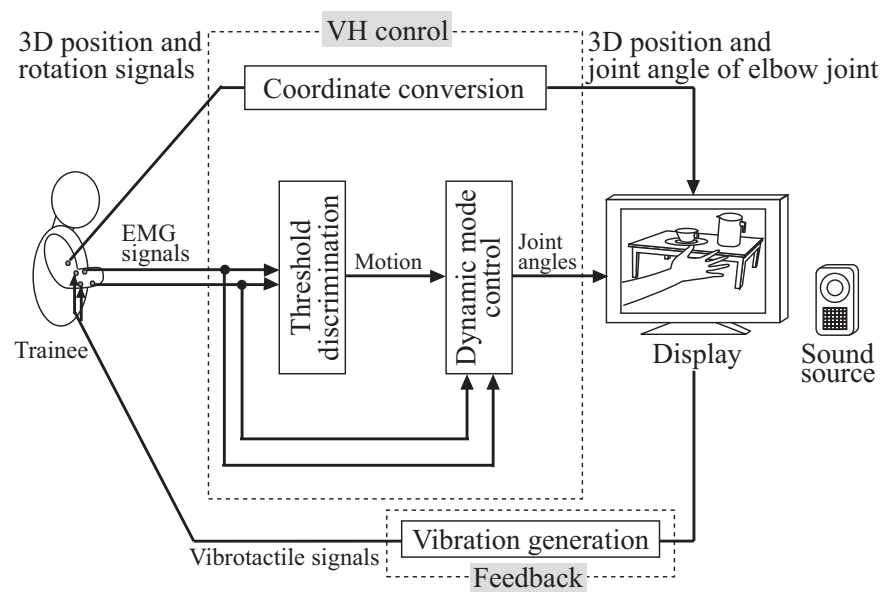

Fig. 1. Structure of the proposed training system

\section{B. Task Training}

Figure 1 shows a structural representation of task training. The trainee can perform tasks such as holding and moving a virtual object using the proposed system.

1) Virtual Hand (VH) and VR Environment: The VH and virtual objects are developed using Virtools [16] that can create and control various virtual objects. The $\mathrm{VH}$ has rigid link structure consists of 22 joints (elbow, wrist and fingers) based on human arm and has human skin-like texture. Each joint of the $\mathrm{VH}$ has three rotation degrees of freedom $[\alpha, \beta, \gamma]^{\mathrm{T}}$ and the elbow joint has three translational degrees of freedom $[x, y, z]^{\mathrm{T}}$. In addition, the system has collision detection between each joint and virtual objects.

2) VH Control: VHs are controlled using EMG signals and position/posture as recorded from the trainee. The details of the processes involved are outlined below.

The opening-closing of a VH is controlled using the MyoBock method. Trainees are first fitted with a socket featuring two built-in electrodes used to record EMG signals. The recorded signals are digitized using an A/D converter (sampling frequency: $\left.f_{s}[\mathrm{~Hz}]\right)$ and are defined as $e_{l}(n)(l \in\{1,2\}$, $n$ : number of samples). The trainee's motion $m \in\{o, c\}$ (o: opening, c: closing) is discriminated based on the amplitude of $e_{l}(n)$ as follows:

$$
m=\left\{\begin{array}{ll}
o & \left(e_{1}\left(n^{\prime}\right) \geq e^{\mathrm{th}}\right) \\
c & \left(e_{2}\left(n^{\prime}\right) \geq e^{\mathrm{th}}\right)
\end{array},\right.
$$

where $n^{\prime}$ is the number of samples observed when $e_{1}(n)$ or $e_{2}(n)$ exceeds the pre-defined threshold $e^{\text {th }}$. The behavior of MyoBock dynamic mode control (DMC) is simulated using the discrimination result $m$ and $e_{l}(n)$ in the proposed system. As the opening/closing angular velocity changes with EMG signal amplitude in the DMC method, the joint angles $\theta(n)$ between the thumb and fingers of the $\mathrm{VH}$ are controlled based on the following equation:

$$
\theta(n)=\theta(n-1)+\omega_{m}\left(e_{l}(n)\right) \Delta t,
$$

where $\omega_{m}\left(e_{l}(n)\right)$ is the opening/closing angular velocity and $\Delta t$ is the sampling time. Here, if the maximum angles for
TABLE I

EXAMPLES OF INITIAL AND MAXIMUM JOINT ANGLES (ALL ANGLES IN DEGREES)

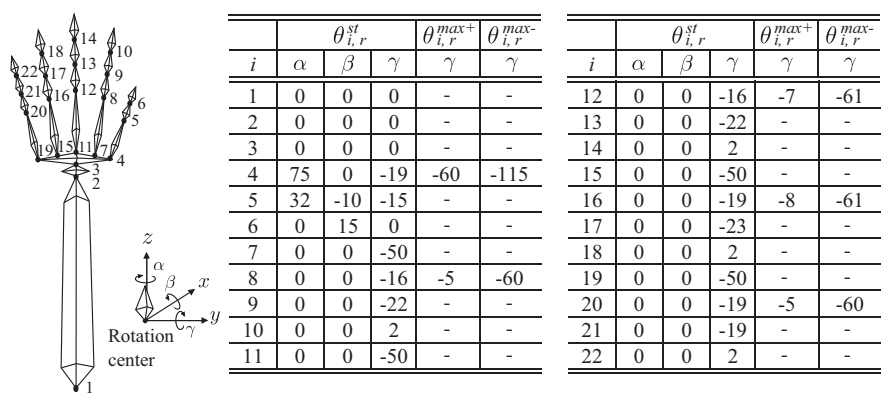

each joint are set as shown in Table 1, pinching motion with MyoBock is realized.

Additionally, the 3D position $\left[x^{\prime}, y^{\prime}, z^{\prime}\right]^{\mathrm{T}}$ and the Eulerian angle $\left[\alpha^{\prime}, \beta^{\prime}, \gamma^{\prime}\right]^{\mathrm{T}}$ recorded from the position sensor attached to the operator's cubital fossa is used for controlling position and posture of the VH. These recorded data based on the right-handed system are converted into the left-handed system because the coordinate system of Virtools is the left-handed one. The 3D position converted into the left-handed system is defined as $[x, y, z]^{\mathrm{T}}$ and the Eulerian angle is defined as $[\alpha, \beta, \gamma]^{\mathrm{T}}$. Moreover, the fingertip position used for detecting gripping objects and the training evaluation is defined as follow:

$$
\boldsymbol{P}_{\text {hand }}(n)=\boldsymbol{P}_{\text {elbow }}(n)+A\left[\begin{array}{c}
\cos \beta^{\prime} \sin \gamma^{\prime} \\
\sin \left(-\beta^{\prime}\right) \\
\cos \beta^{\prime} \cos \gamma^{\prime}
\end{array}\right] \text {, }
$$

where $A$ is a length of a forearm which is configured beforehand.

3) Virtual Feedback: The trainee is provided with auditory and tactile feedback to support the communication of spatial and tactile information in the VR environment. As amputees can perceive vibration from real-environment collision with objects via the socket, the proposed system gives the trainee tactile feedback via vibrotactile stimulators (VBW32C25, Audiological Engineering Corp.) when the $\mathrm{VH}$ touches a virtual object. In this study, vibration of $250 \mathrm{~Hz}$ was used for tactile feedback because humans are the most sensitive to this frequency [17]. Trainees were also provided with auditory feedback when the virtual object was grasped and deposited as intended.

\section{EXPERIMENTS}

In this study, the virtual task training was developed with reference to the Box and Block Test [18] used to evaluate myoelectric prostheses, and a training experiment was conducted. The subjects were two healthy males (A and B). Subject B had experienced EMG control before. Consideration of prosthesis weight was allowed in the training using a socket with the MyoBock developed for attachment to the arms of healthy subjects (Fig. 2). The socket has two built-in electrodes (Otto Bock Corp.; 13E200 = 60; frequency band width: 90-450 


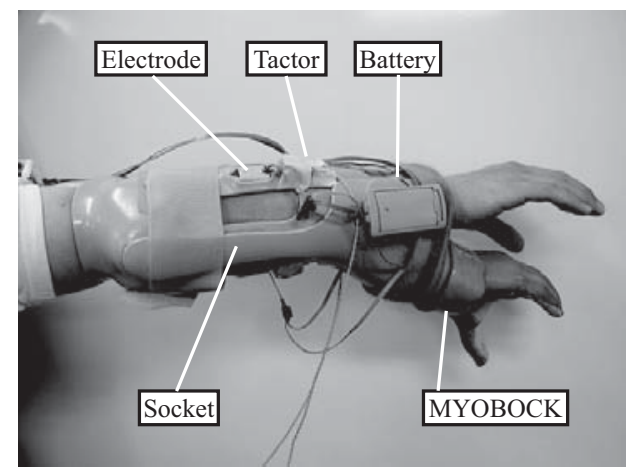

Fig. 2. Socket for attaching the MyoBock to a healthy subject

\begin{tabular}{|c|c|c|}
\hline $\mathrm{BBT}^{*} \times 5$ & Virtual BBT × 10 & $\mathrm{BBT} \times 5$ \\
\hline
\end{tabular}

Fig. 3. Experiment training schedule

[Hz], length: 27 [mm], breadth: $18[\mathrm{~mm}]$, height: 9.5 [mm], weight: $4.5[\mathrm{~g}])$ which were attached to each subject $(L=2$; ch. 1: extensor digitorum muscle; ch. 2: flexor carpi ulnaris muscle) to record EMG signals. This method allows subjects to practice in consideration of the weight of the prosthesis, which is approximately 400 [g] for a MyoBock unit. A 3D position sensor was also attached to each subject's right cubital fossa, and the $3 \mathrm{D}$ position $\left[x^{\prime}, y^{\prime}, z^{\prime}\right]^{\mathrm{T}}$ (accuracy: $\pm 2.4[\mathrm{~mm}]$ ) and the Eulerian angle $\left[\alpha^{\prime}, \beta^{\prime}, \gamma^{\prime}\right]^{\mathrm{T}}$ (accuracy: $\pm 0.75\left[{ }^{\circ}\right]$ ) were recorded. Two vibrotactile stimulators providing tactile feedback were attached to the outer surfaces of the sockets near the electrodes. The parameters used in the proposed system were set as $e^{\text {th }}=0.56[\mathrm{~V}]$ and $f_{s}=1,000[\mathrm{~Hz}]$.

In the experiments with the proposed system, 10 one-minute tests were performed. BBT in a real environment was also conducted to verify the effectiveness of the system. Five sessions of BBT were conducted both before and after the training, whose schedule is outlined in Fig. 3. The subjects' task performance ability was evaluated from the number of blocks transferred in one minute.

\section{A. Box and Block Test in Virtual Reality Environment}

The subjects did not undergo EMG signal control training because they were already able to generate and control EMG signals. Figures 4 and 5 show scenes from training using the proposed system and an example of signals recorded during the 10th session from Subject A. The times shown for the pictures in Fig. 4 correspond to those in Fig. 5. Figure 5 indicate EMG signal discrimination results, position and posture recorded via the $3 \mathrm{D}$ position sensor, the distance between the $\mathrm{VH}$ and the ball $P(n)$, the signal input to the tactile stimulator, and the joint angle between the thumb and index finger $\theta(n)$. The shaded areas represent times during which $e_{l}(n)$ was greater than the pre-defined threshold $e^{\text {th }}$. It can be seen that the trainee received tactile feedback when the VH and the ball collided. The results indicate that Subject A grasped the ball at about $46.9 \mathrm{~s}$, moved it and released it at about $47.9 \mathrm{~s}$, and was able to perform the tasks repeatedly. Figure 6 (a) shows the results of evaluation for successful tasks
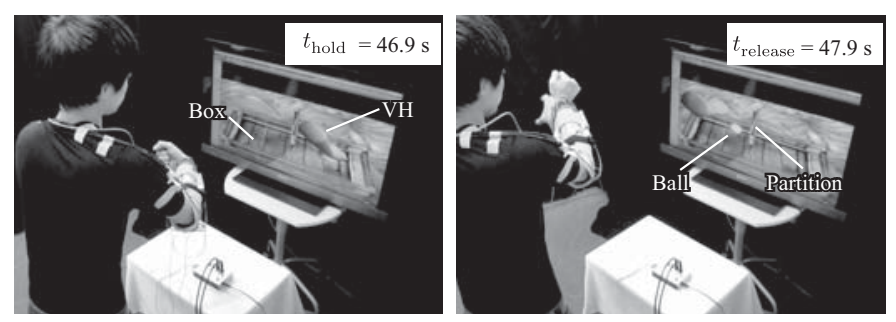

Fig. 4. Operation scenes of the virtual BBT in the final session (Subject A)

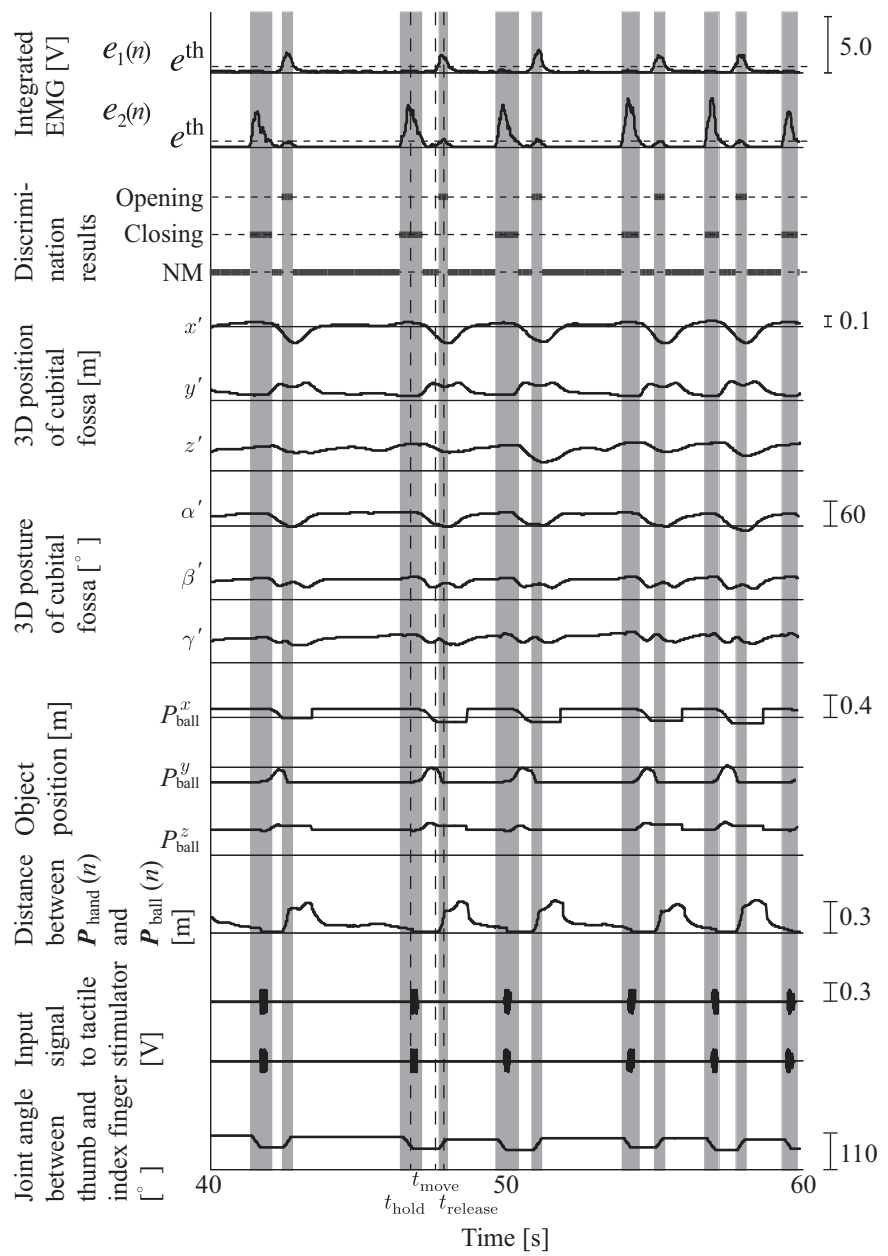

Fig. 5. Example of experimental results from virtual BBT. Subject A repeatedly held, moved and released the virtual object. The shaded areas show the motion generated (NM: no motion).

using the proposed system. It can be seen that the number of successful tasks gradually increased. Here, the values for Subject A were smaller in sessions 7 and 8 . This was because the ball was moved to a location where it was difficult to perceive distance between the hand and balls. Comparing the first and last sessions, the number of successful tasks increased from 11 to 14 for Subject A, and from 17 to 19 for Subject B. These results suggest that the trainees learned to perform more tasks within a given time period using proposed system.

\section{B. Box and Block Test in Real Environment}

Figure 7 shows scenes from BBT and Fig. 6 (b) shows the related results. The circles plotted in Fig. 7 show the base positions of the middle finger at time intervals of $0.3 \mathrm{~s}$. In 


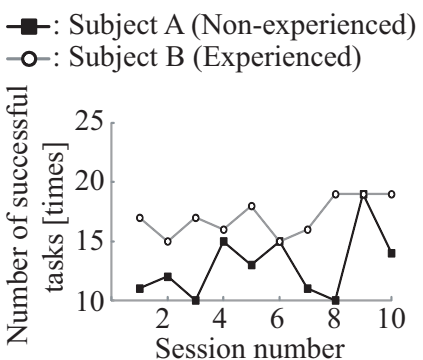

(a) Results of the virtual BBT

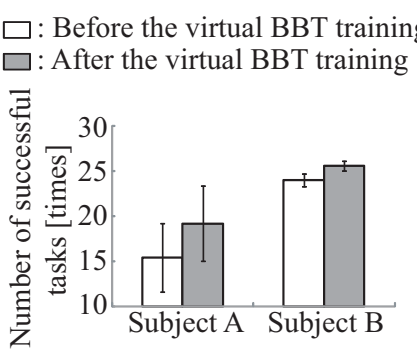

(b) Results of the BBT

Fig. 6. Results of task performance (number of successful tasks) for each subject
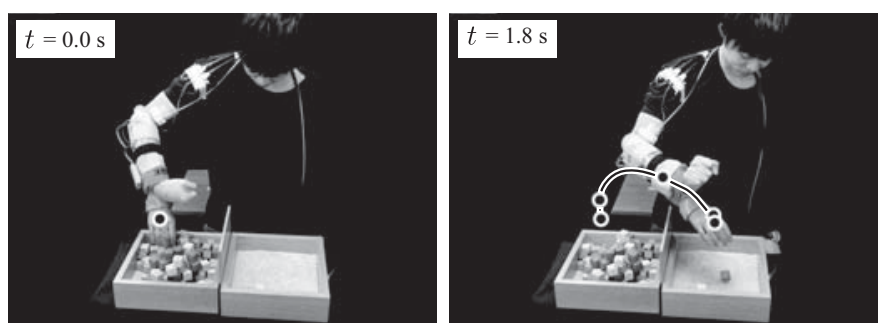

Fig. 7. BBT operation scenes. The plotted data show the base of the MyoBock's middle finger at intervals of $0.3 \mathrm{~s}$.

Fig. 6 (b), the results for subjects A and B both show that the number of successful tasks increased after virtual training. In particular, the number of tasks completed successfully by Subject A approached that of the more experienced Subject B. It is therefore considered that task performance in a real environment was improved by virtual task training using the proposed system. However, as there was no significant difference between the results observed before and after training, the authors plan to check the validity of the proposed system with a longer training period and more subjects.

\section{CONCLUSiON}

This paper proposes an EMG-based virtual training system for MyoBock. In the study, a training experiment was conducted using the proposed system, and BBT was performed in a real environment before and after the virtual training to verify the system's validity. As the results showed that the number of successful tasks increased after the virtual training with the system, it was concluded that the approach can be used for training in myoelectric prosthesis control.

In future research, the authors plan to further verify the validity of the proposed system with a longer training period and more subjects, and improve the proposed system by comparing the differences between the virtual environment and real environment. Other aims include the development of an interactive training system that can display the ideal arm trajectory toward an object for each task, and the establishment of a method for selecting appropriate electrode positions and training motions [19], [20].

\section{ACKNOWLEDGMENT}

This work was supported in part by a Grant-in-Aid for Scientific Research from the Research Fellowships of the Japan

Society for the Promotion of Science for Young Scientists (23.522) and the Japanese Ministry of Education, Science and Culture under Grant 22591634.

\section{REFERENCES}

[1] R. B. Jerard, T. W. Williams and C. W. Ohlenbusch: "Practical Design of an EMG Controlled Above Elbow Prothesis", Proceeding of the 1974 Conference on Engineering Devices in Rehabilitation, pp. 73-77, 1974.

[2] S. C. Jacobson, D. F. Knutti, R.T. Johnson and H. H. Sears: "Development of the Utah Artificial Arm", IEEE Transactions on Biomedical Engineering, vol. 29, no. 4, pp. 249-269, 1982.

[3] O. Fukuda, T. Tsuji, M. Kaneko and A. Otsuka: "A Human-Assisting Manipulator Teleoperated by EMG Signals and Arm Motions", IEEE Transactions on Robotics and Automation, vol. 19, no. 2, pp. 210-222, 2003.

[4] M. C. Carrozza, G. Cappiello, S. Micera, B. B. Edin, L. Beccai and C. Cipriani: "Design of a cybernetic hand for perception and action", Biological Cybernetics, vol. 95, no. 6, pp. 629-644, 2006.

[5] T. Chin: "The training manual of the EMG-based prosthetic hand", All Japan Hospital Publication Association Ltd., pp. 28-30, 2006. (in Japanese)

[6] T. Tsuji, O. Fukuda, O. Akira and K. Makoto: "The EMG controlling training system for the EMG-based prosthetic hand", The institute of Electronics, Information and Communication Engineers, Vol. J83-D-II, 1999. (in Japanese)

[7] O. Fukuda, T. Tsuji, O. Akira and K. Makoto: "An EMG-based Rehabilitation Aid for Prosthetic Control", IEEE Transactions on Robot and Human Communication, vol. 1, pp. 214-219, 2003.

[8] A. C. Dupont and E. L. Morin: "A Myoelectric Control Evaluation and Trainer System", IEEE Transactions on Rehabilitation Engineering, vol. 2, pp. 100-107, 1994.

[9] Otto Bock web page: http://www.ottobock.com/

[10] A. Soares, A. Andrada, E. Lamounier and R. Carrijo: "The Development of a Virtual Myoelectric Prosthesis Controlled by an EMG Pattern Recognition System Based on Neural Networks", Journal of Intelligent Information Systems, vol. 21, no. 2, pp. 127-141, 2003.

[11] O. Fukuda, T. Tsuji, A. Otsuka and M. Kaneko: "A Human Supporting Manipulator Using Neural Network and Its Clinical Application for Forearm Amputation", Proceedings of the 1999 IEEE International Conference on Knowledge-Based Intelligent Information Engineering Systems, pp. 129-134, 1999.

[12] M. Kuttuva, G. Burdea, J. Flint and W. Craelium: "Manipulation Practice for Upper-Limb Amputee Using Virtual Reality", MIT Press Journals, vol. 14 , no. 2 , pp. $175-182,2005$.

[13] T. Takeuchi, T. Wada, M. Mukobaru and S. Doi: "A Training System for Myoelectric Prosthetic Hand in Virtual Environment", IEEE/ICME International Conference on Complex Medical Engineering, pp. 13511356, 2007.

[14] M. Hauschild, R. Davoodi and G. E. Loeb: "A Virtual Reality Environment for Designing and Fitting Neural Prosthetic Limbs", IEEE Transactions on Neural System and Rehabilitation Engineering, vol. 15, no. 1, pp. 9-15, 2007.

[15] J. M. Lambrecht, C.L. Pulliam and R. F. Kirsch: "Virtual Reality Environment for Simulating Tasks With a Myoelectric Prosthesis: An Assessment and Training Tool", American Academy of Orthotists \& Prosthests, vol. 23, no. 2, pp. 89-94, 2011.

[16] Virtools web page: http://www.3ds.com/products/3dvia/3dvia-virtools/

[17] A. Higashiyama: "Tactile sense and pain", Brain Corp., 2000 (in japanese).

[18] V. Mathiowetz, G. Volland N. Kashman and K. Weber: "Adult Norms for the Box and Block Test of Manual Dexterity", The American Journal Occupational Therapy, vol. 39, no. 6, pp. 386-391, 1985.

[19] T. Shibanoki, K. Shima, T. Takaki, Y. Kurita, A. Otsuka, T. Chin and T. Tsuji: "Multi-channel Surface EMG Classification Based on a Quasioptimal Selection of Motions and Channels", IEEE/ICME International Conference on Complex Medical Engineering (CME2012), pp. 276-279, 2012.

[20] T. Shibanoki, K. Shima, T. Toshio, A. Otsuka and T. Chin: "A QuasiOptimal Channel Selection Method for Bioelectric Signal Classification Using a Partial Kullback-Leibler Information Measure", IEEE Transactions on Biomedical Engineering, vol. 60, no. 3, pp. 853-861, 2013. 